\title{
Introduction to Endocrine Disrupting Chemicals - is it time to act?
}

\author{
Christian A. Koch ${ }^{1}$ Evanthia Diamanti-Kandarakis ${ }^{2,3}$
}

Published online: 17 February 2016

(C) Springer Science+Business Media New York 2016

Endocrine Disrupting Chemicals (EDCs) are considered as an emerging public health issue due to their potency to interfere and deregulate several aspects of the endocrine system physiology in laboratory animals, wildlife, and humans. Human exposure is thought to be constant and universal and has been related with diverse perturbations including reproductive disorders, thyroid and metabolic dysfunction, hormone-dependent cancers and neurodevelopmental diseases.

Substantial evidence incriminates endocrine disruptors as key players in the increasing incidence of modern human disorders raising awareness among and by the scientific community and public policies over their adverse effects. In response to these concerns, this Guest issue intends to provide all recent data connecting endocrine disruptors and endocrine health/disorders applying the knowledge of prominent scientists working in this field.

It is a great honor to introduce guest editor and expert consultant, Professor (Prof.) Evanthia Diamanti-Kandarakis, who previously chaired an Endocrine Society scientific statement on EDCs, and has recruited an outstanding group of contributing authors. Each one of them has provided an

Evanthia Diamanti-Kandarakis

e.diamanti.kandarakis@gmail.com

Christian A. Koch

ckoch@umc.edu

1 Division of Endocrinology, Head, Endocrine Tumor Program, Cancer Institute, University of Mississippi Medical Center, Clin Sci Bldg, Rm 512, 2500 N State Street, Jackson, MS 39216, USA

2 Athens University Medical School, Athens, Greece

3 Athens - Euroclinic, 9 Athanasiadou, 115-21 Athens, Greece excellent review of very important and contemporary topics related to EDCs.

Prof. Vandenberg and colleague analyzed the crucial issue of methodologies been developed to identify the hazard and assess the risk of endocrine disruptors. This is particularly important in the evaluation of scientific evidence for decision making. Facilitating the understanding of methodological issues in human studies of EDCs is further emphasized by Prof. Jacobs and colleague who focused on the assessment of human exposure studies of EDCs which combined with the aforementioned review article provides insights into the methodological arguments of human data evaluation.

Next, the connection between EDCs, specifically bisphenol A (BPA) and the global epidemic of obesity, metabolic syndrome and type 2 diabetes, has been explored by Prof. Fenichel and colleague.

Another interesting issue analyzed by Prof. Duntas and colleague is the potential deleterious involvement of environmental pollutants on normal thyroid function through direct toxic effect on several stages of thyroid regulation. Expanding this topic further into autoimmune thyroid disorders and differentiated thyroid cancer, Prof. Benvenga and colleagues reviewed the experimental and clinical literature on effects of the environmental and occupational exposure to polluting chemicals, providing tables with thus far known geographic locations and populations.

From a clinical perspective, Prof. Sonnenschein and colleagues present evidence linking exposure to environmental contaminants with incidences of altered development and neoplasia of male reproductive organs. A similar approach to the female reproductive system and mainly carcinogenesis is provided by Prof. Rachon, explaining the mechanisms involved in EDCs-related development of estrogen dependent cancer. 
Guest Editor Prof. Diamanti-Kandarakis and colleague analyzed evidence linking BPA with the pathogenesis of polycystic ovary syndrome.

Finally, the section of "femininity" and EDCs includes data presented by Prof. Sasco and colleagues illuminating the role of the cosmetics industry as an alternative source of exposure to these chemicals.
In conclusion, this Guest issue provides an up-to-date review of the current literature linking endocrine disruptors and human health starting from methods applied for data assessment and expanding to the clinical aspects of endocrine disruption in humans. 